\title{
Asymptotic analysis of the Hermite polynomials from their differential-difference equation
}

\author{
Diego Dominici * \\ Department of Mathematics \\ State University of New York at New Paltz \\ 75 S. Manheim Blvd. Suite 9 \\ New Paltz, NY 12561-2443 \\ USA \\ Phone: (845) 257-2607 \\ Fax: (845) 257-3571
}

February 2, 2008

\begin{abstract}
We analyze the Hermite polynomials $H_{n}(x)$ and their zeros asymptotically, as $n \rightarrow \infty$. We obtain asymptotic approximations from the differential-difference equation which they satisfy, using the ray method. We give numerical examples showing the accuracy of our formulas.
\end{abstract}

Keywords: Hermite polynomials, asymptotic analysis, ray method, orthogonal polynomials, differential-difference equations, discrete WKB method. MSC-class: 33C45 (Primary) 34E05, 34E20 (Secondary)

*e-mail: dominicd@newpaltz.edu 


\section{Introduction}

It would be difficult to find a more ubiquitous polynomial family than the Hermite polynomials $H_{n}(x)$, defined by the Rodrigues formula

$$
H_{n}(x)=(-1)^{n} \exp \left(x^{2}\right) \frac{d^{n}}{d x^{n}} \exp \left(-x^{2}\right), \quad n=0,1,2, \ldots
$$

They appear in several problems of mathematical physics 23, the most important probably being the solution of the Schrödinger equation [6], 14]. Being the limiting case of several families of classical orthogonal polynomials [16, they are of fundamental importance in asymptotic analysis [24], 33].

The Hermite polynomials satisfy the orthogonality condition

$$
\int_{-\infty}^{\infty} e^{-x^{2}} H_{m}(x) H_{n}(x) d x=\sqrt{\pi} 2^{n} n ! \delta_{m n},
$$

the differential-difference equation

$$
H_{n+1}+H_{n}^{\prime}=2 x H_{n}
$$

and the reflection formula

$$
H_{n}(-x)=(-1)^{n} H_{n}(x) .
$$

The zeros of the Hermite polynomials have several applications, notably in Gauss' quadrature formula for numerical integration [13, [29], [30. Several properties and their asymptotic behavior were studied in [1], 2], 3], 4], 7], [26], 28, 31 and 32.

The asymptotic behavior of $H_{n}(x)$ was studied by M. Plancherel and W. Rotach in [27] using the method that now bears their name. F. W. J. Olver 25] obtained asymptotic expansions for the Hermite polynomials as a consequence of his WKB analysis of the differential equation satisfied by the Parabolic Cylinder function $D_{\nu}(z)$, related to $H_{n}(x)$ by

$$
H_{n}(x)=2^{\frac{n}{2}} \exp \left(\frac{x^{2}}{2}\right) D_{n}(\sqrt{2} x)
$$

A similar analysis using perturbation techniques was carried on by A. Voznyuk in 34]. 
As an application of the results from his doctoral thesis on the multiplicationinterpolation method, L. Heflinger [15] established asymptotic series for the Hermite polynomials. In [39, M. Wyman derived asymptotic formulas for $H_{n}(x)$ based on one of their integral representations.

In this paper we shall take a different approach and analyze the differentialdifference equations that the Hermite polynomials satisfy (11) using the techniques presented in [10]. A similar method (which we may call the discrete WKB method) has been applied to the solution of difference equations [5], [8, 12, 38, and it is currently being extended [11, 35, 36, 37, to include difference equations with turning points. Another type of analysis, based on perturbation techniques, was considered by C. Lange and R. Miura in [17, [18], 19], 20], 21], and [22].

\section{Asymptotic analysis}

We consider the approximation

$$
H_{n}(x) \sim \exp [f(x, n)+g(x, n)], \quad n \rightarrow \infty
$$

where

$$
g=o(f), \quad n \rightarrow \infty .
$$

Note that since $H_{0}(x)=1$, we must have

$$
f(x, 0)=0
$$

and

$$
g(x, 0)=0
$$

Using (3) in (11), we have

$$
\begin{gathered}
\exp \left(f+\frac{\partial f}{\partial n}+\frac{1}{2} \frac{\partial^{2} f}{\partial n^{2}}+g+\frac{\partial g}{\partial n}\right) \\
+\left(\frac{\partial f}{\partial x}+\frac{\partial g}{\partial x}\right) \exp (f+g)=2 x \exp (f+g),
\end{gathered}
$$

where we have used

$$
f(x, n+1)=f(x, n)+\frac{\partial f}{\partial n}(x, n)+\frac{1}{2} \frac{\partial^{2} f}{\partial n^{2}}(x, n)+\cdots .
$$


Simplifying (17) and taking (4) into account we obtain, to leading order,

$$
\exp \left(\frac{\partial f}{\partial n}\right)+\frac{\partial f}{\partial x}=2 x .
$$

Using (8) in (71) we get

$$
\exp \left(\frac{1}{2} \frac{\partial^{2} f}{\partial n^{2}}+\frac{\partial g}{\partial n}\right)+\frac{\partial g}{\partial x} \exp \left(-\frac{\partial f}{\partial n}\right)=1,
$$

or, to leading order,

$$
\frac{1}{2} \frac{\partial^{2} f}{\partial n^{2}}+\frac{\partial g}{\partial n}+\frac{\partial g}{\partial x} \exp \left(-\frac{\partial f}{\partial n}\right)=0 .
$$

\subsection{The ray expansion}

To solve (8) we use the method of characteristics, which we briefly review. Given the first order partial differential equation

$$
F(x, n, f, p, q)=0
$$

where

$$
p=\frac{\partial f}{\partial x}, \quad q=\frac{\partial f}{\partial n},
$$

we search for a solution $f(x, n)$ by solving the system of "characteristic equations"

$$
\begin{aligned}
& \frac{d x}{d t}=\frac{\partial F}{\partial p}, \quad \frac{d n}{d t}=\frac{\partial F}{\partial q} \\
& \frac{d p}{d t}=-\frac{\partial F}{\partial x}-p \frac{\partial F}{\partial f}, \quad \frac{d q}{d t}=-\frac{\partial F}{\partial n}-q \frac{\partial F}{\partial f} \\
& \frac{d f}{d t}=p \frac{\partial F}{\partial p}+q \frac{\partial F}{\partial q}
\end{aligned}
$$

where we now consider $\{x, n, f, p, q\}$ to all be functions of the variables $t$ and $s$.

For (18), we have

$$
F(x, n, f, p, q)=e^{q}+p-2 x
$$


and therefore the characteristic equations are

$$
\frac{d x}{d t}=1, \quad \frac{d n}{d t}=e^{q}, \quad \frac{d p}{d t}=2, \quad \frac{d q}{d t}=0,
$$

and

$$
\frac{d f}{d t}=p+q e^{q}
$$

Solving (11) subject to the initial conditions

$$
x(0, s)=s, \quad n(0, s)=0, \quad q(0, s)=A(s),
$$

we obtain

$$
x=t+s, \quad n=t e^{A}, \quad p=2 t+2 s-e^{A}, \quad q=A,
$$

where we have used

$$
0=\left.F\right|_{t=0}=e^{A}+p(0, s)-2 s .
$$

From (15) and (13) we have

$$
f(0, s)=0,
$$

which implies

$$
\begin{aligned}
0 & =\frac{d}{d s} f(0, s)=\left[\frac{\partial f}{\partial x} \frac{\partial x}{\partial s}+\frac{\partial f}{\partial n} \frac{\partial n}{\partial s}\right]_{t=0} \\
& =p(0, s) \times 1+q(0, s) \times 0=2 s-e^{A} .
\end{aligned}
$$

Thus, $A(s)=\ln (2 s)$ and (14) becomes

$$
x=t+s, \quad n=2 t s, \quad p=2 t, \quad q=\ln (2 s),
$$

with $t \geq 0$ and $s>0$. Since $s>0$, we shall consider only the region $x>0$ for now. Using (16) in (12) and taking (15) into account, we obtain

$$
f(t, s)=t^{2}+2 s \ln (2 s) t .
$$

Solving for $t$ and $s$ in terms of $x$ and $n$ in (16), we get

$$
t=\frac{x}{2} \pm \frac{1}{2} \sigma, \quad s=\frac{x}{2} \mp \frac{1}{2} \sigma
$$


with

$$
\sigma=\sqrt{x^{2}-2 n}
$$

For $\sigma$ to be a real number, we shall impose the condition $x>\sqrt{2 n}$. Since (for a fixed value of $n$ ) we have $t \rightarrow 0$ as $x \rightarrow \infty$, we consider the solution

$$
t=\frac{x}{2}-\frac{1}{2} \sigma, \quad s=\frac{x}{2}+\frac{1}{2} \sigma .
$$

Replacing (20) in (17) we obtain

$$
f(x, n)=\frac{x^{2}-\sigma x-n}{2}+n \ln (x+\sigma), \quad x>\sqrt{2 n} .
$$

We shall now find $g(x, n)$. Using (21) in (9), we get

$$
-\frac{1}{2 \sigma(x+\sigma)}+\frac{\partial g}{\partial n}+\frac{\partial g}{\partial x} \frac{1}{(x+\sigma)}=0,
$$

or

$$
(x+\sigma) \frac{\partial g}{\partial n}+\frac{\partial g}{\partial x}=\frac{1}{2 \sigma} .
$$

Solving (22), we obtain

$$
g(x, n)=\frac{1}{2} \ln \left(-2 \frac{x^{2}-n+x \sigma}{x^{2}-2 n+x \sigma}\right)+C(x+\sigma),
$$

where $C(x)$ is a function to be determined. Imposing the condition (6), we have

$$
0=g(x, 0)=\frac{1}{2} \ln (-2)+C(2 x) .
$$

Thus,

$$
g(x, n)=\frac{1}{2} \ln \left[\frac{1}{2}\left(\frac{x}{\sigma}+1\right)\right] .
$$

We summarize our results in the following theorem.

Theorem 1 In the region $x>\sqrt{2 n}$, the Hermite polynomials admit the asymptotic representation

$$
\begin{aligned}
H_{n}(x) \sim \Phi_{1}(x, n)= & \exp \left[\frac{x^{2}-\sigma x-n}{2}+n \ln (\sigma+x)\right] \\
& \times \sqrt{\frac{1}{2}\left(1+\frac{x}{\sigma}\right)}, \quad n \rightarrow \infty,
\end{aligned}
$$

where $\sigma(x, n)$ was defined in (19). 


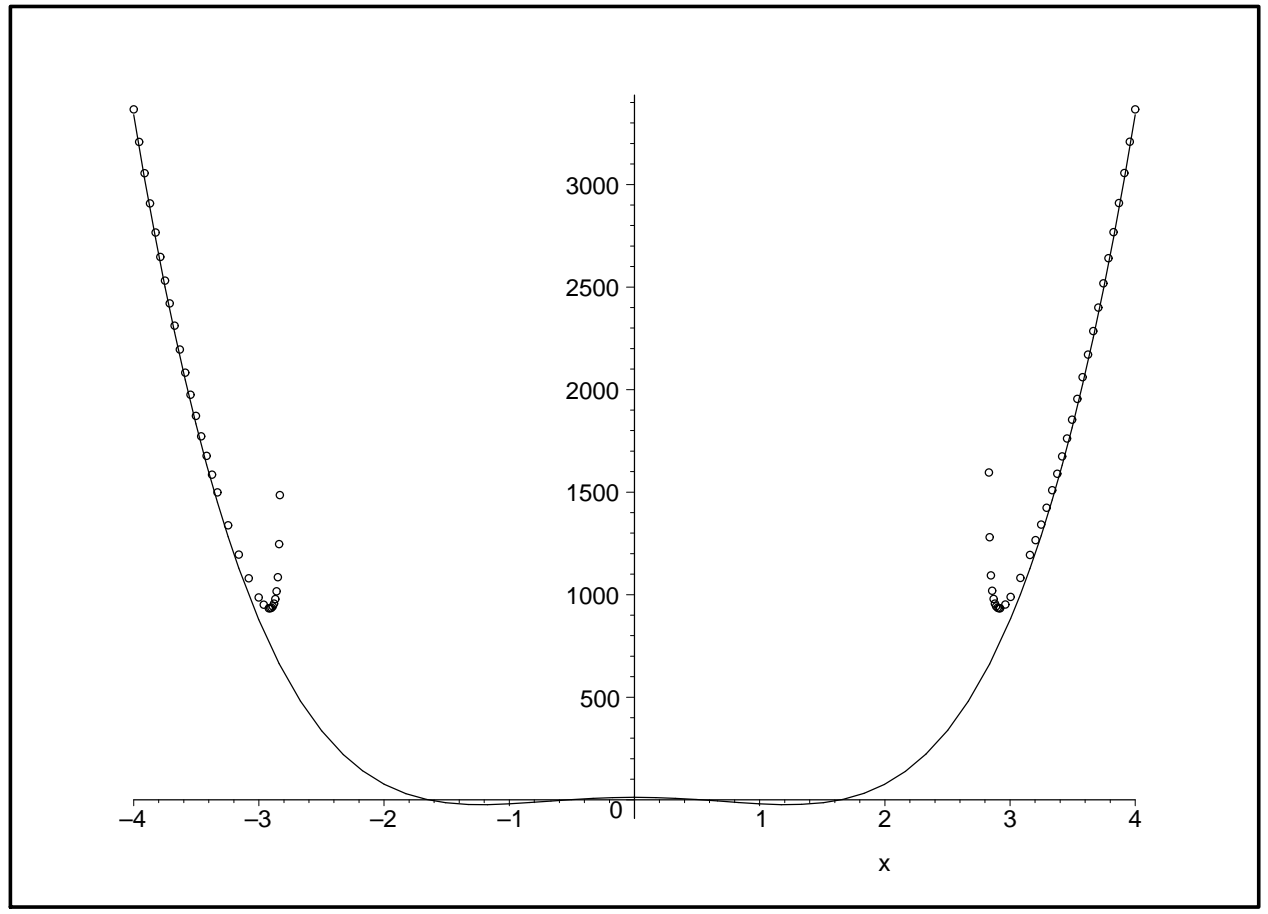

Figure 1: A comparison of $H_{4}(x)$ (solid curve) and the asymptotic approximations $\Phi_{1}(x, 4)$ and $\Phi_{2}(x, 4)$ (ooo).

Using the reflection formula (2) we can extend our result to the region $-x>\sqrt{2 n}$ and obtain:

Corollary 2 In the region $x<-\sqrt{2 n}$, the Hermite polynomials admit the asymptotic representation

$$
\begin{aligned}
H_{n}(x) \sim \Phi_{2}(x, n)= & (-1)^{n} \exp \left[\frac{x^{2}+\sigma x-n}{2}+n \ln (\sigma-x)\right] \\
& \times \sqrt{\frac{1}{2}\left(1-\frac{x}{\sigma}\right)}, \quad n \rightarrow \infty .
\end{aligned}
$$

To illustrate the accuracy of our results, in Figure 1 we graph $H_{4}(x)$ and the asymptotic approximations $\Phi_{1}(x, 4)$ and $\Phi_{2}(x, 4)$. 


\subsection{The transition layer}

We shall now find an asymptotic approximation for $|x| \approx \sqrt{2 n}$. We will consider the case $x \approx \sqrt{2 n}$ and find the corresponding result for $x \approx-\sqrt{2 n}$ by using (2). From (24) we have

$$
\Phi_{1}(x, n) \sim \exp \left[\frac{n}{2} \ln (2 n)-\frac{3}{2} n+\sqrt{2 n} x\right], \quad x \longrightarrow \sqrt{2 n}^{+} .
$$

We define the function $G_{n}(x)$ by

$$
H_{n}(x)=\exp \left[\frac{n}{2} \ln (2 n)-\frac{3}{2} n+\sqrt{2 n} x\right] G_{n}(x) .
$$

Using (26) in (11) we get

$$
\begin{aligned}
& \exp \left[\frac{n+1}{2} \ln (2 n+2)\right.\left.-\frac{n}{2} \ln (2 n)-\frac{3}{2}+(\sqrt{2(n+1)}-\sqrt{2 n}) x\right] G_{n+1} \\
&+\sqrt{2 n} G_{n}+G_{n}^{\prime}=2 x G_{n}(x) .
\end{aligned}
$$

We introduce the stretch variable $\beta>0$ defined by

$$
x=\sqrt{2 n}+\frac{\beta}{n^{\frac{1}{6}}}
$$

and the function $\Lambda(\beta)$ defined by

$$
G_{n}(x)=\Lambda\left[(x-\sqrt{2 n}) n^{\frac{1}{6}}\right] .
$$

From (28) we have

$$
\begin{aligned}
& \exp \left[\frac{n+1}{2} \ln (2 n+2)\right.\left.-\frac{n}{2} \ln (2 n)-\frac{3}{2}+(\sqrt{2(n+1)}-\sqrt{2 n}) x\right] \\
& \sim \sqrt{2 n}+\beta n^{-\frac{1}{6}}, \quad n \rightarrow \infty
\end{aligned}
$$

Using (28) in (29) we obtain

$$
\begin{aligned}
G_{n+1}(x) & =\Lambda\left[\left(\sqrt{2 n}-\sqrt{2 n+1}+\frac{\beta}{n^{\frac{1}{6}}}\right)(n+1)^{\frac{1}{6}}\right] \\
& \sim \Lambda(\beta)-\frac{1}{\sqrt{2}} \Lambda^{\prime}(\beta) n^{-\frac{1}{3}}+\frac{1}{4} \Lambda^{\prime \prime}(\beta) n^{-\frac{2}{3}}, \quad n \rightarrow \infty,
\end{aligned}
$$


and

$$
\sqrt{2 n} G_{n}+G_{n}^{\prime}-2 x G_{n}(x)=-\sqrt{2 n} \Lambda(\beta)+\Lambda^{\prime}(\beta) n^{\frac{1}{6}}-2 \beta \Lambda(\beta) n^{-\frac{1}{6}} .
$$

Using (30), (31) and (32) in (27) we obtain, to leading order, the Airy equation

$$
\Lambda^{\prime \prime}(\beta)=2 \sqrt{2} \beta \Lambda(\beta)
$$

Thus,

$$
\Lambda(\beta)=C_{1} \operatorname{Ai}(\sqrt{2} \beta)+C_{2} \operatorname{Bi}(\sqrt{2} \beta),
$$

where $\mathrm{Ai}(\cdot)$ and $\mathrm{Bi}(\cdot)$ denote the Airy functions and $C_{1}, C_{2}$ are to be determined. Replacing (28) and (34) in (26) we have

$$
H_{n}(x) \sim \exp \left[\frac{n}{2} \ln (2 n e)+\sqrt{2} \beta n^{\frac{1}{3}}\right]\left[C_{1} \mathrm{Ai}(\sqrt{2} \beta)+C_{2} \operatorname{Bi}(\sqrt{2} \beta)\right] .
$$

To find $C_{1}, C_{2}$ we shall match (35) with (24). Using (28) in (24) we get

$$
\Phi_{1}(x, n) \sim \exp \left[\frac{n}{2} \ln (2 n e)+\sqrt{2} \beta n^{\frac{1}{3}}-\frac{2^{\frac{7}{4}}}{3} \beta^{\frac{3}{2}}\right] 2^{-\frac{5}{8}} \beta^{-\frac{1}{4}} n^{\frac{1}{6}},
$$

as $\beta \rightarrow 0$. Using (28) and the well known asymptotic expansions of the Airy functions

$$
\begin{aligned}
& \mathrm{Ai}(x) \sim \frac{1}{2 \sqrt{\pi}} \exp \left(-\frac{2}{3} x^{\frac{3}{2}}\right) x^{-\frac{1}{4}}, \quad x \rightarrow \infty \\
& \mathrm{Bi}(x) \sim \frac{1}{\sqrt{\pi}} \exp \left(\frac{2}{3} x^{\frac{3}{2}}\right) x^{-\frac{1}{4}}, \quad x \rightarrow \infty,
\end{aligned}
$$

in (35) we have

$$
\exp \left[\frac{n}{2} \ln (2 n)-\frac{3}{2} n+\sqrt{2 n} x\right]=\exp \left\{\frac{n}{2}[1+\ln (2 n)]+\sqrt{2} \beta n^{\frac{1}{3}}\right\}
$$

and

$$
\begin{gathered}
C_{1} \operatorname{Ai}(\sqrt{2} \beta)+C_{2} \operatorname{Bi}(\sqrt{2} \beta) \sim \\
\frac{C_{1}}{\sqrt{\pi} 2^{\frac{9}{8} \beta^{\frac{1}{4}}}} \exp \left(-\frac{2^{\frac{7}{4}}}{3} \beta^{\frac{3}{2}}\right)+\frac{C_{2}}{\sqrt{\pi} 2^{\frac{1}{8}} \beta^{\frac{1}{4}}} \exp \left(\frac{2^{\frac{7}{4}}}{3} \beta^{\frac{3}{2}}\right), \quad \beta \rightarrow \infty .
\end{gathered}
$$


Matching (36) to (37) and (38), we conclude that

$$
C_{1}=\sqrt{2 \pi} n^{\frac{1}{6}}, \quad C_{2}=0 .
$$

This completes the analysis. Combining the results above, we have the following result:

Theorem 3 For $x \approx \sqrt{2 n}$, the Hermite polynomials have the asymptotic representation

$$
\begin{aligned}
H_{n}(x) & \sim \Phi_{3}(x, n)=\exp \left[\frac{n}{2} \ln (2 n)-\frac{3}{2} n+\sqrt{2 n} x\right] \\
& \times \sqrt{2 \pi} n^{\frac{1}{6}} \text { Ai }\left[\sqrt{2}(x-\sqrt{2 n}) n^{\frac{1}{6}}\right], \quad n \rightarrow \infty .
\end{aligned}
$$

Use of the reflection formula (21) provides the corresponding result for $x \approx-\sqrt{2 n}$.

Corollary 4 For $x \approx-\sqrt{2 n}$, the Hermite polynomials have the asymptotic representation

$$
\begin{aligned}
H_{n}(x) & \sim \Phi_{4}(x, n)=(-1)^{n} \exp \left[\frac{n}{2} \ln (2 n)-\frac{3}{2} n-\sqrt{2 n} x\right] \\
& \times \sqrt{2 \pi} n^{\frac{1}{6}} \mathrm{Ai}\left[-\sqrt{2}(x+\sqrt{2 n}) n^{\frac{1}{6}}\right], \quad n \rightarrow \infty .
\end{aligned}
$$

\subsection{The oscillatory region}

We now study the region bounded by the curve $n=\frac{x^{2}}{2}$, where the zeros of $H_{n}(x)$ are located. In this region, the solution is a linear combination of (24) and (25)

$$
H_{n}(x) \sim \Phi_{5}(x, n) \equiv K_{1} \Phi_{1}(x, n)+K_{2} \Phi_{2}(x, n), \quad n \rightarrow \infty
$$

with $|x|<\sqrt{2 n}$ and $K_{1}, K_{2}$ are constants to be determined. We shall require $\Phi_{5}(x, n)$ to match $\Phi_{3}(x, n)$ asymptotically in the local variable $\beta$, i.e., it must satisfy the limiting condition

$$
\lim _{\beta \rightarrow 0} \Phi_{5}(\beta, n)=\lim _{\beta \rightarrow-\infty} \Phi_{3}(\beta, n) .
$$


Writing (40) in terms of $\beta$, we have

$$
\Phi_{3}(\beta, n)=\exp \left[\frac{n}{2} \ln (2 n e)+\sqrt{2} \beta n^{\frac{1}{3}}\right] \sqrt{2 \pi} n^{\frac{1}{6}} \mathrm{Ai}(\sqrt{2} \beta) .
$$

Using the asymptotic formula

$$
\operatorname{Ai}(x) \sim \frac{1}{\sqrt{\pi}} \sin \left[\frac{2}{3}(-x)^{\frac{3}{2}}+\frac{\pi}{4}\right](-x)^{-\frac{1}{4}}, \quad x \rightarrow-\infty
$$

in (42) we get

$$
\begin{aligned}
& \Phi_{3}(\beta, n) \sim \exp \left[\frac{n}{2} \ln (2 n e)+\sqrt{2} \beta n^{\frac{1}{3}}\right] 2^{\frac{3}{8}} n^{\frac{1}{6}} \\
& \times \sin \left[\frac{1}{3} 2^{\frac{7}{4}}(-\beta)^{\frac{3}{2}}+\frac{\pi}{4}\right](-\beta)^{-\frac{1}{4}}, \quad \beta \rightarrow-\infty,
\end{aligned}
$$

which can be rewritten as

$$
\begin{aligned}
& \Phi_{3}(\beta, n) \sim 2^{-\frac{5}{8}} n^{\frac{1}{6}} \beta^{-\frac{1}{4}} \exp \left[\frac{n}{2} \ln (2 n e)+\sqrt{2} \beta n^{\frac{1}{3}}\right] \\
\times & {\left[\exp \left(-\frac{1}{3} 2^{\frac{7}{4}} \beta^{\frac{3}{2}}\right)+\mathrm{i} \exp \left(\frac{1}{3} 2^{\frac{7}{4}} \beta^{\frac{3}{2}}\right)\right], \quad \beta \rightarrow-\infty . }
\end{aligned}
$$

Using (28) in (24), we have

$$
\Phi_{1}(\beta, n) \sim \exp \left[\frac{n}{2} \ln (2 n e)+\sqrt{2} \beta n^{\frac{1}{3}}-\frac{1}{3} 2^{\frac{7}{4}} \beta^{\frac{3}{2}}\right] 2^{-\frac{5}{8}} n^{\frac{1}{6}} \beta^{-\frac{1}{4}}, \quad \beta \rightarrow 0 .
$$

Similarly, using (28) in (25), we obtain

$$
\Phi_{2}(\beta, n) \sim \exp \left[\frac{n}{2} \ln (2 n e)+\sqrt{2} \beta n^{\frac{1}{3}}+\frac{1}{3} 2^{\frac{7}{4}} \beta^{\frac{3}{2}}\right] 2^{-\frac{5}{8}} n^{\frac{1}{6}} \beta^{-\frac{1}{4}} \mathrm{i}, \quad \beta \rightarrow 0,
$$

where we have used

$$
(-1)^{n} \exp \left[\frac{x^{2}+\sigma x-n}{2}+n \ln (\sigma-x)\right]=\exp \left[\frac{x^{2}+\sigma x-n}{2}+n \ln (x-\sigma)\right] \text {. }
$$

Comparing (43) with (44) and (45) we conclude that $K_{1}=1=K_{2}$ and therefore

$$
\Phi_{5}(x, n)=\Phi_{1}(x, n)+\Phi_{2}(x, n) .
$$


Since $-\sqrt{2 n}<x<\sqrt{2 n}$, we set

$$
x=\sqrt{2 n} \sin (\theta), \quad-\frac{\pi}{2}<\theta<\frac{\pi}{2} .
$$

Using (47) in (19), we have

$$
\sigma=\sqrt{2 n} \cos (\theta) \mathrm{i}
$$

Replacing (48) in (24), we get

$$
\begin{gathered}
\exp \left[\frac{x^{2}-\sigma x-n}{2}+n \ln (\sigma+x)\right]= \\
\exp \left\{\frac{n}{2}[\ln (2 n)-\cos (2 \theta)]-n\left[\frac{1}{2} \sin (2 \theta)+\theta-\frac{\pi}{2}\right] \mathrm{i}\right\}
\end{gathered}
$$

and

$$
\sqrt{\frac{1}{2}\left(1+\frac{x}{\sigma}\right)}=\frac{\exp \left(-\frac{\theta}{2} \mathrm{i}\right)}{\sqrt{2 \cos (\theta)}} .
$$

Similarly, replacing (48) in (25), we obtain

$$
\begin{gathered}
(-1)^{n} \exp \left[\frac{x^{2}+\sigma x-n}{2}+n \ln (\sigma-x)\right]= \\
\exp \left\{\frac{n}{2}[\ln (2 n)-\cos (2 \theta)]+n\left[\frac{1}{2} \sin (2 \theta)+\theta-\frac{\pi}{2}\right] \mathrm{i}\right\}
\end{gathered}
$$

and

$$
\sqrt{\frac{1}{2}\left(1-\frac{x}{\sigma}\right)}=\frac{\exp \left(\frac{\theta}{2} \mathrm{i}\right)}{\sqrt{2 \cos (\theta)}} .
$$

Using (49)-(152) in (46), we have

$$
\begin{gathered}
\Phi_{5}[\sqrt{2 n} \sin (\theta), n]=\sqrt{\frac{2}{\cos (\theta)}} \exp \left\{\frac{n}{2}[\ln (2 n)-\cos (2 \theta)]\right\} \\
\times \cos \left\{n\left[\frac{1}{2} \sin (2 \theta)+\theta-\frac{\pi}{2}\right]+\frac{\theta}{2}\right\}, \quad-\frac{\pi}{2}<\theta<\frac{\pi}{2} .
\end{gathered}
$$

Thus, we have proved the following: 
Theorem 5 In the region $|x|<\sqrt{2 n}$, the Hermite polynomials have the asymptotic representation

$$
\begin{gathered}
H_{n}[\sqrt{2 n} \sin (\theta)] \sim \sqrt{\frac{2}{\cos (\theta)}} \exp \left\{\frac{n}{2}[\ln (2 n)-\cos (2 \theta)]\right\} \\
\times \cos \left\{n\left[\frac{1}{2} \sin (2 \theta)+\theta-\frac{\pi}{2}\right]+\frac{\theta}{2}\right\}, \quad n \rightarrow \infty,
\end{gathered}
$$

with $-\frac{\pi}{2}<\theta<\frac{\pi}{2}$.

In Figure 2 we graph

$$
H_{n}[\sqrt{2 n} \sin (\theta)] \exp \left\{-\frac{n}{2}[\ln (2 n)-\cos (2 \theta)]\right\}
$$

and

$$
\sqrt{\frac{2}{\cos (\theta)}} \cos \left\{n\left[\frac{1}{2} \sin (2 \theta)+\theta-\frac{\pi}{2}\right]+\frac{\theta}{2}\right\},
$$

with $n=20$. We only include the range $0 \leq \theta<\frac{\pi}{2}$, since both functions are even.

The same results obtained in this section were derived in [9] using a different method, based on the limit relation between the Charlier and Hermite polynomials [16].

\section{Zeros}

We shall now find asymptotic formulas for the zeros of the Hermite polynomials using the results from the previous section. Let's denote by $\zeta_{1}^{n}>$ $\zeta_{2}^{n}>\cdots>\zeta_{n}^{n}$ the zeros of $H_{n}(x)$, enumerated in decreasing order. Then, it follows from (54) that

$$
\zeta_{k}^{n} \sim \sqrt{2 n} \sin \left(\tau_{k}^{n}\right), \quad n \rightarrow \infty
$$

where $\tau_{k}^{n}$ is a solution of the equation

$$
n\left[\frac{1}{2} \sin \left(2 \tau_{k}^{n}\right)+\tau_{k}^{n}-\frac{\pi}{2}\right]+\frac{\tau_{k}^{n}}{2}=(1-2 k) \frac{\pi}{2}, \quad 1 \leq k \leq n .
$$




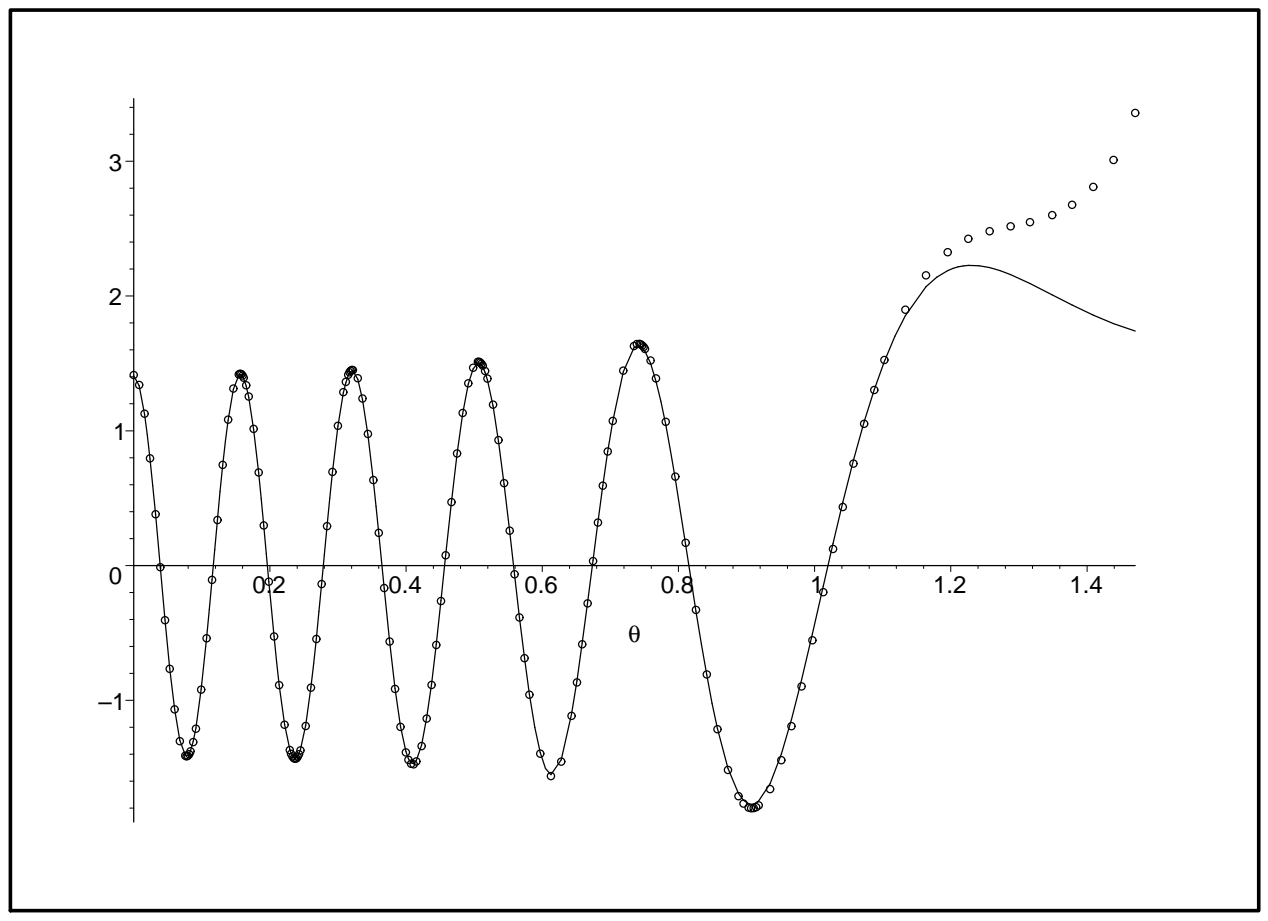

Figure 2: A comparison of the exact (solid curve) and asymptotic (ooo) values of $H_{20}(x)$ in the oscillatory region. 
Solving (56) numerically and using (55) we get very good approximations of $\zeta_{k}^{n}$. One could also solve (56) exactly (as we did in [9]) and obtain a Kapteyn series expansion for $\tau_{k}^{n}$

$$
\tau_{k}^{n}=\frac{\pi}{2}-\frac{\pi}{2}(4 k-1) N^{-1}-\sum_{j=1}^{\infty} \frac{1}{j} \mathrm{~J}_{j}\left[\left(1-N^{-1}\right) j\right] \sin \left(\frac{4 k-1}{N} j \pi\right),
$$

where $N=2 n+1$ and $\mathrm{J}_{j}(\cdot)$ denotes the Bessel function of the first kind. However, (57) is difficult to analyze asymptotically. Hence, we will take a different approach and find an approximation for $\tau_{k}^{n}$ from (56) trough perturbation techniques.

We will consider two cases: $k=O(1)$ which corresponds to the largest zeros of $H_{n}(x)$ and $k=O\left(\frac{n}{2}\right)$, related to the zeros close to $x=0$.

\subsection{Case I: $k=O(1)$}

Replacing

$$
\tau_{k}^{n}=\frac{\pi}{2}-\sum_{i \geq 1} a_{i}(k) n^{-\frac{i}{3}}
$$

in (56) we obtain, as $n \rightarrow \infty$

$$
\begin{aligned}
a_{1} & =\frac{1}{2} \kappa^{\frac{1}{3}}, \quad a_{2}=-\frac{1}{2} \kappa^{-\frac{1}{3}}, \quad a_{3}=\frac{\kappa}{120}, \quad a_{4}=-\frac{\kappa^{-\frac{5}{3}}}{30}\left(\kappa^{2}-5\right) \\
a_{5} & =\frac{\kappa^{-\frac{7}{3}}}{8400}\left(3 \kappa^{4}+350 \kappa^{2}+1400\right), \quad a_{6}=-\frac{43}{16800} \kappa, \\
a_{7} & =\frac{\kappa^{-\frac{11}{3}}}{50400}\left(\kappa^{6}+350 \kappa^{4}-980 \kappa^{2}-11200\right), \\
a_{8} & =-\frac{\kappa^{-\frac{13}{3}}}{63000}\left(13 \kappa^{6}+475 \kappa^{4}+1400 \kappa^{2}+17500\right), \\
a_{9} & =\frac{59}{67200} \kappa+\frac{43}{34496000} \kappa^{3}, \\
a_{10} & =-\frac{\kappa^{-\frac{17}{3}}}{1397088000}\left(23817 \kappa^{8}+2608760 \kappa^{6}-4592280 \kappa^{4}\right. \\
& \left.-51744000 \kappa^{2}-664048000\right),
\end{aligned}
$$

with

$$
\kappa(k)=3 \pi(4 k-1)
$$


Using (158)-(159) in (155), we get

$$
\begin{gathered}
\zeta_{k}^{n} \sim \sqrt{2}\left(n^{\frac{1}{2}}-\frac{\kappa^{\frac{2}{3}}}{8} n^{-\frac{1}{6}}+\frac{1}{4} n^{-\frac{1}{2}}-\frac{\kappa^{2}+80}{640 \kappa^{\frac{2}{3}}} n^{-\frac{5}{6}}\right. \\
-\frac{11 \kappa^{2}+3920}{179200} n^{-\frac{3}{2}}+\frac{5 \kappa^{4}+96 \kappa^{2}+640}{7680 \kappa^{\frac{8}{3}}} n^{-\frac{11}{6}} \\
-\frac{823 \kappa^{6}+647200 \kappa^{4}-2464000 \kappa^{2}-25088000}{258048000 \kappa^{\frac{10}{3}}} n^{-\frac{13}{6}} \\
\left.+\frac{3064+33 \kappa^{2}}{716800} n^{-\frac{5}{2}}\right), \quad n \rightarrow \infty .
\end{gathered}
$$

\subsection{Case II: $k=O\left(\frac{n}{2}\right)$}

We now set

$$
k=\left\lfloor\frac{n}{2}\right\rfloor+1-j=\frac{n}{2}-\alpha+1-j,
$$

where $\alpha=\operatorname{frac}\left(\frac{n}{2}\right)$ (the fractional part of $\frac{n}{2}$ ) and $j=0,1,2, \ldots$ Using (62) and

$$
\tau_{k}^{n}=\sum_{i \geq 1} b_{i}(j) n^{-i}
$$

in (56) we obtain, as $n \rightarrow \infty$

$$
\begin{aligned}
& b_{1}=\xi, \quad b_{2}=-\frac{\xi}{4}, \quad b_{3}=\frac{\xi}{48}\left(3+16 \xi^{2}\right), \\
& b_{4}=-\frac{\xi}{192}\left(3+64 \xi^{2}\right), \quad b_{5}=\frac{\xi}{3840}\left(15+800 \xi^{2}+1024 \xi^{4}\right), \\
& b_{6}=-\frac{\xi}{15360}\left(15+1600 \xi^{2}+7424 \xi^{4}\right),
\end{aligned}
$$

with

$$
\xi(j)=\frac{\pi}{4}(2 j+2 \alpha-1) .
$$

Using (63)-(64) in (55), we obtain

$$
\zeta_{k}^{n} \sim \sqrt{2} \xi\left(n^{-\frac{1}{2}}-\frac{1}{4} n^{-\frac{3}{2}}+\frac{3+8 \xi^{2}}{48} n^{-\frac{5}{2}}-\frac{3+80 \xi^{2}}{192} n^{-\frac{7}{2}}\right), \quad n \rightarrow \infty .
$$

In Table1 we compare the exact value of the positive zeros of $H_{20}(x)$ with the approximations given by solving (56) numerically and formulas (61) and 
Table 1: A comparison of the exact and approximate values for the positive zeros of $H_{20}(x)$.

\begin{tabular}{|c|c|c|c|}
\hline$\zeta_{k}^{n}$ & $(\overline{566})$ & $\overline{(666)}$ & ([61) \\
\hline .24534 & .24536 & .24536 & - \\
\hline .73747 & .73751 & .73750 & - \\
\hline 1.2341 & 1.2342 & 1.2340 & - \\
\hline 1.7385 & 1.7387 & 1.7376 & - \\
\hline 2.2550 & 2.2552 & 2.2512 & 2.2592 \\
\hline 2.7888 & 2.7892 & 2.7779 & 2.7912 \\
\hline 3.3479 & 3.3486 & - & 3.3492 \\
\hline 3.9448 & 3.9456 & - & 3.9460 \\
\hline 4.6037 & 4.6056 & - & 4.6055 \\
\hline 5.3875 & 5.3939 & - & 5.3937 \\
\hline
\end{tabular}

(66). Note that the biggest error corresponds to the larger zero, where the asymptotic approximation (154) almost breaks down.

We summarize the results of this section in the following theorem.

Theorem 6 Letting $\zeta_{1}^{n}>\zeta_{2}^{n}>\cdots>\zeta_{n}^{n}$ be the zeros of $H_{n}(x)$, enumerated in decreasing order, we have:

1.

$$
\zeta_{k}^{n} \sim \sqrt{2}\left(n^{\frac{1}{2}}-\frac{\kappa^{\frac{2}{3}}}{8} n^{-\frac{1}{6}}+\frac{1}{4} n^{-\frac{1}{2}}-\frac{\kappa^{2}+80}{640 \kappa^{\frac{2}{3}}} n^{-\frac{5}{6}}\right), \quad n \rightarrow \infty,
$$

where $k=O(1)$ and $\kappa(k)$ was defined in $(60)$.

2.

$$
\zeta_{k}^{n} \sim \sqrt{2} \xi\left(n^{-\frac{1}{2}}-\frac{1}{4} n^{-\frac{3}{2}}+\frac{3+8 \xi^{2}}{48} n^{-\frac{5}{2}}-\frac{3+80 \xi^{2}}{192} n^{-\frac{7}{2}}\right), \quad n \rightarrow \infty,
$$

where $k=\frac{n}{2}-\alpha+1-j, \alpha=\operatorname{frac}\left(\frac{n}{2}\right)$ and $\xi(j)$ was defined in (65)). 


\section{References}

[1] S. Ahmed. Systems of nonlinear equations for the zeros of Hermite polynomials. Lett. Nuovo Cimento (2), 22(9):367-370, 1978.

[2] I. Area, D. K. Dimitrov, E. Godoy, and A. Ronveaux. Zeros of Gegenbauer and Hermite polynomials and connection coefficients. Math. Comp., 73(248):1937-1951 (electronic), 2004.

[3] F. Calogero. On the zeros of Hermite polynomials. Lett. Nuovo Cimento (2), 20(14):489-490, 1977.

[4] F. Calogero and A. M. Perelomov. Asymptotic density of the zeros of Hermite polynomials of diverging order, and related properties of certain singular integral operators. Lett. Nuovo Cimento (2), 23(18):650-652, 1978.

[5] O. Costin and R. Costin. Rigorous WKB for finite-order linear recurrence relations with smooth coefficients. SIAM J. Math. Anal., 27(1):110-134, 1996.

[6] J. S. Dehesa, F. Domínguez-Adame, E. R. Arriola, and A. Zarzo. Hydrogen atom and orthogonal polynomials. In Orthogonal polynomials and their applications (Erice, 1990), volume 9 of IMACS Ann. Comput. Appl. Math., pages 223-229. Baltzer, Basel, 1991.

[7] H. Dette and W. J. Studden. Some new asymptotic properties for the zeros of Jacobi, Laguerre, and Hermite polynomials. Constr. Approx., 11(2):227-238, 1995.

[8] R. B. Dingle and G. J. Morgan. WKB methods for difference equations. I, II. Appl. Sci. Res., 18:221-237; 238-245, 1967/1968.

[9] D. Dominici. Asymptotic analysis of the Askey-scheme II: from Charlier to Hermite. Submitted, 2005. arXiv: math.CA/0508264.

[10] T. Dosdale, G. Duggan, and G. J. Morgan. Asymptotic solutions to differential-difference equations. J. Phys. A, 7:1017-1026, 1974.

[11] J. S. Geronimo, O. Bruno, and W. Van Assche. WKB and turning point theory for second-order difference equations. In Spectral methods 
for operators of mathematical physics, volume 154 of Oper. Theory Adv. Appl., pages 101-138. Birkhäuser, Basel, 2004.

[12] J. S. Geronimo and D. T. Smith. WKB (Liouville-Green) analysis of second order difference equations and applications. J. Approx. Theory, 69(3):269-301, 1992.

[13] R. E. Greenwood and J. J. Miller. Zeros of the Hermite polynomials and weights for Gauss' mechanical quadrature formula. Bull. Amer. Math. Soc., 54:765-769, 1948.

[14] K. Hannabuss. An introduction to quantum theory, volume 1 of Oxford Graduate Texts in Mathematics. The Clarendon Press Oxford University Press, New York, 1997.

[15] L. O. Heflinger. The asymptotic behaviour of the Hermite polynomials. Nederl. Akad. Wetensch. Proc. Ser. A. 59 = Indag. Math., 18:255-264, 1956.

[16] R. Koekoek and R. F. Swarttouw. The Askey-scheme of hypergeometric orthogonal polynomials and its $q$-analogue. Technical Report 98-17, Delft University of Technology, 1998. http://aw.twi.tudelft.nl koekoek/askey/.

[17] C. G. Lange and R. M. Miura. Singular perturbation analysis of boundary value problems for differential-difference equations. SIAM J. Appl. Math., 42(3):502-531, 1982.

[18] C. G. Lange and R. M. Miura. Singular perturbation analysis of boundary value problems for differential-difference equations. II. Rapid oscillations and resonances. SIAM J. Appl. Math., 45(5):687-707, 1985.

[19] C. G. Lange and R. M. Miura. Singular perturbation analysis of boundary value problems for differential-difference equations. III. Turning point problems. SIAM J. Appl. Math., 45(5):708-734, 1985.

[20] C. G. Lange and R. M. Miura. Singular perturbation analysis of boundary value problems for differential-difference equations. IV. A nonlinear example with layer behavior. Stud. Appl. Math., 84(3):231-273, 1991. 
[21] C. G. Lange and R. M. Miura. Singular perturbation analysis of boundary value problems for differential-difference equations. V. Small shifts with layer behavior. SIAM J. Appl. Math., 54(1):249-272, 1994.

[22] C. G. Lange and R. M. Miura. Singular perturbation analysis of boundary value problems for differential-difference equations. VI. Small shifts with rapid oscillations. SIAM J. Appl. Math., 54(1):273-283, 1994.

[23] N. N. Lebedev. Special functions and their applications. Dover Publications Inc., New York, 1972.

[24] J. L. López and N. M. Temme. Hermite polynomials in asymptotic representations of generalized Bernoulli, Euler, Bessel, and Buchholz polynomials. J. Math. Anal. Appl., 239(2):457-477, 1999.

[25] F. W. J. Olver. Uniform asymptotic expansions for Weber parabolic cylinder functions of large orders. J. Res. Nat. Bur. Standards Sect. B, 63B:131-169, 1959.

[26] G. Pittaluga and L. Sacripante. Bounds for the zeros of Hermite polynomials. Ann. Numer. Math., 2(1-4):371-379, 1995. Special functions (Torino, 1993).

[27] M. Plancherel and W. Rotach. Sur les valeurs asymptotiques des polynomes d'Hermite $H_{n}(x)=(-I)^{n} e^{\frac{x^{2}}{2}} \frac{d^{n}}{d x^{n}}\left(e^{-\frac{x^{2}}{2}}\right)$. Comment. Math. Helv., 1(1):227-254, 1929.

[28] P. E. Ricci. Improving the asymptotics for the greatest zeros of Hermite polynomials. Comput. Math. Appl., 30(3-6):409-416, 1995.

[29] J. B. Rosser. Note on zeros of the Hermite polynomials and weights for Gauss' mechanical quadrature formula. Proc. Amer. Math. Soc., 1:388-389, 1950.

[30] H. E. Salzer, R. Zucker, and R. Capuano. Table of the zeros and weight factors of the first twenty Hermite polynomials. J. Research Nat. Bur. Standards, 48:111-116, 1952.

[31] P. R. Subramanian. Zeros of the Hermite polynomials and the simple Laguerre polynomials are irrational. In Proceedings of the Third Annual 
Conference of Vijnana Parishad of India and the National Symposium of Ancient Science in India (Srinagar, 1993), volume 1, pages 49-57, 1993.

[32] P. R. Subramanian. Nonzero zeros of the Hermite polynomials are irrational. Fibonacci Quart., 33(2):131-134, 1995.

[33] N. M. Temme and J. L. López. The role of Hermite polynomials in asymptotic analysis. In Special functions (Hong Kong, 1999), pages 339-350. World Sci. Publishing, River Edge, NJ, 2000.

[34] A. V. Voznyuk. Asymptotic formulas for parabolic cylindrical functions and for Hermite polynomials for large values of the argument. Vychisl. Prikl. Mat. (Kiev), (63):19-24, 125, 1987.

[35] Z. Wang and R. Wong. Uniform asymptotic expansion of $J_{\nu}(\nu a)$ via a difference equation. Numer. Math., 91(1):147-193, 2002.

[36] Z. Wang and R. Wong. Asymptotic expansions for second-order linear difference equations with a turning point. Numer. Math., 94(1):147-194, 2003.

[37] Z. Wang and R. Wong. Linear difference equations with transition points. Math. Comp., 74(250):629-653 (electronic), 2005.

[38] P. Wilmott. A note on the WKB method for difference equations. IMA J. Appl. Math., 34(3):295-302, 1985.

[39] M. Wyman. The asymptotic behaviour of the Hermite polynomials. Canad. J. Math., 15:332-349, 1963. 